\title{
VALORES O VALORACIONES. APORTES DE LA ANTROPOLOGÍA A LA INVESTIGACIÓN EN BIOÉTICA
}

\author{
Nelson Eduardo Álvarez Licona*
}

Resumen: Este artículo analiza el aporte de la antropología a la bioética, en cuanto a su metodología y a sus marcos teóricos de referencia. Sostiene que las mayores confusiones provienen de la aplicación mecánica de los así llamados "valores universales"; éstas se disolverían si, cuando se interpreta el comportamiento moral, se comprende que en realidad no estamos frente a valores, sino a valoraciones.

Palabras clave: dignidad, identidad, valores, valoraciones, metodologías, técnicas de investigación

\section{VALUES OR APPRAISEMENTS. ANTHROPOLOGY'S CONTRIBUTIONS IN BIOETHICS RESEARCH}

Abstract: This paper analyzes anthropology's contribution to bioethics, related to its methodology and theoretical frameworks of reference. It affirms that most confusions arise from the mechanic application of the so called "universal values" which would be solved if, when interpreting moral behavior, we understood that we are not considering values but appraisements..

Key words: dignity, identity, values, assessment, methodology, research techniques

\section{VALORES OU VALORIZAÇÓES: CONTRIBUIÇÁO DA ANTROPOLOGIA PARA A PESQUISA EM BIOÉTICA}

Resumo: Este artigo analisa a contribuição da antropologia para a bioética, no que toca à sua metodologia e seus marcos teóricos de referencia. Sustenta que as maiores confusões surgem da aplicação mecânica dos assim chamados "valores universais". Estas confusóes se dissolvem, quando se interpreta o comportamento moral, se compreende que na realidade não estamos frente a valores mas, a valorizaçốes.

Palavras-chave: dignidade, identidade, valores, valorizaçôes, metodologias, técnicas de pesquisa

Doctor en Antropología Social. Sección de Estudios de Postgrado e Investigación, Escuela Superior de Medicina, Instituto Politécnico Nacional, México

Correspondencia: nalvarez@ipn.mx 


\section{Introducción}

Uno de los principales problemas a los que se enfrenta el estudio y la práctica de la bioética es la aplicación mecánica de lo que se ha dado en llamar "valores universales". La creencia en éstos se derrumba cuando nos percatamos de que siempre estamos en presencia de valoraciones que parten de contextos determinados espacio-temporalmente. El análisis de una cultura nos permite entender ese contexto y, además, el comportamiento de los sujetos que lo comparten, lo que no implica estar de acuerdo con su explicación de la realidad.

Aceptar la existencia de valores universales importa reconocer algo que está más allá del hombre, que trasciende el espacio y el tiempo. Sin embargo, nunca nos encontramos, por ejemplo, ante la libertad, sino ante una valoración respecto de ciertas prácticas. Si partimos de la convicción de que los valores son entidades con existencia propia, no quedaría más que la aplicación mecánica de sus preceptos, dejando a la experiencia humana al margen de su necesaria contextualización espacio-temporal. La argumentación de la existencia de valores como entidades suprahumanas, encarnadas en el hombre, se ilustra en afirmaciones como la siguiente: "Estoy muy lejos de la arrogancia escéptica con que a menudo se trata a los valores. Me son también ajenas las tendencias (que se dan frecuentemente) de 'reducirlos' a cualquier modo subjetivo de comportamiento del hombre o de comunidades humanas enteras, como si no hubiera los valores mismos y únicamente hubiera ciertos engaños e ilusiones que resultan de ciertas actitudes y necesidades individuales o que han adquirido carta de naturaleza en algunas comunidades"(1).

Un ejemplo de las implicaciones que plantea el tratamiento de los valores desde las dos perspectivas es el de la "dignidad humana" que se desprende del concepto "persona”, gestado en el pensamiento cristiano desde la reflexión sobre el misterio trinitario y su aplicación a la misma persona de Cristo. En los primeros siglos del cristianismo transitó desde la teología hasta la filosofía, refiriéndose primero al carácter trinitario divino para luego aludir a la existencia de Dios en el hombre. Lo que dará lugar en la legislación de Roma a la designación de un sujeto de derechos y deberes, dejando la significación indeterminada que aludía a cualquier objeto, como era entendido por los griegos, para referirse a un sujeto regulado mediante las leyes de la ciudad. A partir de aquí se comienza a debatir acerca del sentido de "persona" (diferenciándola de "sustancia"), que implicaba la presencia de Dios. Para los romanos, prósôpon no designará un objeto sino a un sujeto personal, a un ser espiritual con una "dignidad" constitutiva de su mismo ser(2). La dignidad es, entonces, irrenunciable, es innata al hombre. No puede ser demostrada, ya que depende de la aceptación, mediante la fe, de la existencia de Dios.

Existe, en contraposición, otra corriente de pensamiento que ubica la categoría "dignidad" en relación con la práctica social y no con lo ontológico. Esta forma de conceptualizarla la encontramos en la definición del Diccionario de la Lengua Española: "excelencia, realce // Gravedad y decoro de las personas en la manera de comportarse // Cargo o empleo honorífico y de autoridad”. Como decoro, Gómez Pin, apunta: "una primera connotación del término que en este trabajo nos ocupa, la dignidad como decencia, en el sentido de la adecuación a la mirada del otro, que sólo asténicamente se conserva en el uso habitual del término 'decoro'" (3).

La palabra se relaciona entonces con la valoración del comportamiento moral desde la mirada exterior. La dignidad es atribuida desde un juicio y no tiene una explicación ontológica, sino que requiere para su comprensión del referente espacio-temporal de la cultura, en la cual encontramos los elementos que nos permiten construir una interpretación de lo que percibimos. $\mathrm{Al}$ percibir formalizamos y es a partir de esa forma con que damos sentido a lo que percibimos que podemos estar ante realidades conocidas. La formalización depende de los criterios de interpretación aprendidos que corresponden a las estructuras propias de la sociedad. "El hombre es un animal colgado de una red de significado que él mismo ha tejido. Para mí la cultura son esas redes y su análisis no debe ser una ciencia experimental en búsqueda de leyes, sino una ciencia interpretativa en búsqueda de significado"(4).

Una cultura determinada establece lo permitido y lo no permitido. "Cada cultura descartaba elementos que eran incongruentes, modificaba otros para sus propios fines e inventaba otros más que coincidieran con sus gustos. El resultado era una forma de vida dispuesta en torno a unos cuantos principios estéticos e intelectuales que producían una cosmovisión. La tarea del antropólogo no es tan sólo registrar una mirada de detalles acerca de un pueblo, sino demostrar una unidad más profunda que integra los diferentes rasgos de una cultura"(4). 
Dentro de la cultura se gestan los valores mediante los cuales se valida el comportamiento social, formando así los criterios de verdad y los juicios de valor, partes de las estructuras que permiten entender la práctica social, al ser principios generadores y organizadores de las prácticas y sus representaciones(5).

La argumentación respecto de la dignidad no es una discusión inocente, ni exclusivamente académica, sino que refleja la explicación de la realidad desde presupuestos teóricos que en este caso son contrapuestos, encontrándonos con que está en juego no sólo la explicación de un concepto, sino la implementación de políticas de control social, como es el caso de los grupos que plantean, desde el argumento ontológico, la defensa de la vida bajo cualquier circunstancia. Basados en este supuesto, sostienen que la dignidad es constitutiva del ser humano; que todo hombre ha sido concebido divinamente al serle infundida su dignidad, pues es manifestación de Dios mismo, mediante la hipóstasis que se acepta como inmanente al ser humano. La relevancia de aceptar la propuesta indemostrable del origen óntico de la dignidad se muestra en la creación de la categoría de "digneidad" para referirse a la "dignidad ontológica, premoral, de la persona, como ser digno por el solo hecho de ser persona e, incluso, al margen de su comportamiento moral" $(2, p .165)$. Al no poder negar la existencia del referente espacio-temporal, se ha concebido la existencia de la categoría "digneidad" sin negar a la primera. Este nuevo concepto sólo puede ser aceptado si se parte del supuesto de la existencia de Dios, mediante la fe. Con este tipo de argumentos se pretende crear las condiciones para que las propuestas de explicación se conviertan en prácticas sociales: aceptación de los criterios de verdad impuestos sin cuestionamientos, que conviertan estas explicaciones en principios de actuación y en regulación de prácticas sociales.

En los juicios morales encontramos referentes que sólo se explican a través de la sociedad como generadora de la identidad definida como "estado de realidad". Entiendo por tal el momento de nuestra vida en el cual estamos siendo. En la concreción de nuestro existir nos encontramos con la condición de que, a la vez que encarnación de la sociedad que nos proporciona los contextos, formalizamos en una existencia propia la realidad desde nuestra óptica particular. La identidad es una experiencia constante en la concreción de nuestro existir. Vivimos un proceso de permanente cambio, en el cual los diferentes elementos que nos conforman inciden en nuestra percepción e interpretación de la realidad, dependiendo de qué tan importante es su presencia en un momento determinado. Por ejemplo, nuestros estados de ánimo pueden determinar la percepción estética e, incluso, cambiar el sentido de comunicación que un gesto nos provoque.

La reflexión acerca del comportamiento moral conlleva necesariamente la consideración de los valores. Si hablamos de valores universales, estamos entonces ante valores eternos. De los valores universales sólo podemos afirmar que han sido una búsqueda constante de la humanidad, pero lo eterno, al estar al margen del espacio y el tiempo, no es posible formalizarlo. Siempre damos forma en base a contextos, pues nuestro entender e interpretar depende de nuestra cultura.

La valoración moral consiste en evaluar con base en criterios de bien y mal. De dichas valoraciones no es posible deducir una obligación universal, sino sólo una conclusión estadística. Cualquier afirmación tácita, individual o grupal, es efímera, pasajera, lo que no se contrapone con el hecho de que la dignidad, la justicia o la libertad haya que respetarlas como acuerdos sociales, al margen de las contingencias ideológicas que sufren nuestras sociedades.

Las valoraciones pueden llevarnos al reconocimiento estadístico de una práctica moral, pero nunca a que la interpretemos como un hecho verdadero, ya que el comportamiento moral no puede tener el aval de la certidumbre científica.

\section{La antropología y su aporte metodológico a la investigación en bioética}

Alfonso Pérez Argote señala que: "el análisis de los fenómenos sociales de identidad colectiva debe hacerse (...) a través de un doble momento metodológico. Un momento (...) en el que se acepta la identidad, la creencia, el sentimiento, la conciencia en cuestión, su definición de la realidad y se observa cómo determina el comportamiento; este momento garantiza la inexistencia de un juicio sobre la cientificidad de la imagen de la realidad, lo que constituiría un auténtico juicio de valor. El momento genético garantiza la consideración de la imagen como variable dependiente, acercándonos a sus determinaciones objetivas y a los mecanismos sociales de producción y reproducción de la imagen. Este doble momento nos acerca a la arbitrariedad 
lógica y a la determinación social de todo fenómeno social"(6).

La antropología aporta la metodología de investigación apropiada para acercarnos al fenómeno u objeto de estudio, de manera que la observación se encuentre lo mejor controlada para evitar los sesgos propios de la persona -juicios de valor y los criterios de cientificidad-, ya que mediante la observación participante se puede penetrar a la interioridad y pasar así de la observación, que discurre en la superficie, a la información en que se encuentra la lógica que sustenta las prácticas. En la Introducción a "Los Argonautas del Pacífico Occidental", Bronislaw Malinowski habla de las condiciones en que debe realizarse el trabajo etnográfico: "lo fundamental es apartarse de la compañía de los otros blancos y permanecer con los indígenas en un contacto tan estrecho como se pueda, lo cual sólo es realmente posible si se acampa en sus mismos poblados"(7). Sólo podemos conocer una cultura o explicar ciertos comportamientos morales adoptando sus propios puntos de vista, lo que no quiere decir compartirlos, sino entender la lógica de explicación en que se sustentan. Un aporte importante de la antropología a la bioética es la observación participante, ya que permite acceder a la información desde las lógicas que la sustentan. Aunque no podemos ignorar que el trabajo de campo descansa tanto en los presupuestos teóricos como en las valoraciones respecto del comportamiento estudiado(8).

Los marcos teóricos que proporcionan la teoría antropológica y las ciencias sociales permiten un acercamiento a ese momento genético apuntado por Pérez Argote y que corresponde al momento étic ${ }^{1}$, condición necesaria para pasar de la descripción a la explicación del fenómeno social que se estudia. Después de hablar de la importancia de una sólida formación científica del etnógrafo, Malinowski apunta una observación fundamental: "No me extenderé más sobre este tema, salvo en una observación para evitar cualquier posible equívoco. Tener una buena preparación teórica y estar al tanto de los datos más recientes no es lo mismo que estar cargado de 'ideas preconcebidas'. Si alguien emprende una expedición decidido a probar determinada hipótesis y es incapaz de cambiar en cualquier momento sus puntos

1 "étic y émic: Distingue entre la comprensión de las representaciones culturales desde el punto de vista de un nativo de la cultura (émic) y la comprensión de las representaciones culturales desde el punto de vista de un observador externo a la cultura (étic)". Barfield T, Schussheim V. Diccionario de Antropología. México: Siglo Veintiuno Editores; 2000. de vista y de desecharlos de buena gana bajo el peso de las evidencias, no hace falta decir que su trabajo no tendrá ningún valor. Cuantos más problemas se plantee sobre la marcha, cuanto más se acostumbre a amoldar sus teorías a los hechos y ver los datos como capaces de configurar una teoría, mejor equipado estará para su trabajo. Las ideas preconcebidas son perniciosas en todo trabajo científico, pero las conjeturas son el don principal de un pensador científico, y tales conjeturas le son posibles al observador sólo gracias a sus estudios teóricos" (7, p. 26).

Si todo conocimiento tiene su origen en la observación y ésta se compone de cuatro elementos: el observador, los fenómenos observados, la información buscada y el papel del observador(9), en el caso de la investigación en bioética, a semejanza de la antropológica, el investigador constituye un elemento decisivo. Es fundamental que el acercamiento a los fenómenos se haga tomando conciencia de y asumiendo la carga moral y los juicios de valor que son parte de la forma como interpretamos lo que percibimos. Por ello, es relevante el aporte de la antropología a la investigación en bioética, mediante los controles clásicos que existen sobre la investigación antropológica. De hecho, algunos de éstos no son sino lógica coincidencia de los procedimientos empleados en ambas disciplinas:

- Controles sobre la ecuación personal, siendo el trabajo en equipo la forma ideal para realizarlo. Los comités de bioética funcionan sobre la base del trabajo en equipo, que además es multidisciplinario, lo que controla los sesgos provocados por las valoraciones de cada miembro en particular.

- Controles sobre la obtención y fiabilidad de los datos. Metodológicamente y mediante el cuaderno de campo, se puede hacer una estricta separación de los tipos de fuentes de datos.

- Contrastar la información ha sido una técnica comúnmente empleada en antropología. Para la investigación en bioética resulta valiosa la contrastación cruzada de la información.

- La falseabilidad, la replicabilidad, la fiabilidad intersubjetiva y la navaja de Occam son principios que se incluyen en la lógica de la ciencia. "Lo que distingue a la ciencia de la pseudociencia no es el tema que se trate, sino la metodología que se emplea" (10).

- Controles sobre los diseños y métodos de investigación. 
La antropología también puede aportar a la formación de bioeticistas la elaboración de programas de formación de observador, para reducir los sesgos, donde se contemplen:

- Datos de sociabilidad del observador.

- Su capacidad de adaptación a situaciones nuevas o impactantes.

- Rigidez en el mantenimiento de sus propios esquemas.

- Reconocimiento de si el sujeto atribuye a causas internas o externas las consecuencias de sus actos.

Con esto se ayuda a que el investigador pueda controlar la influencia de las características de su personalidad sobre el proceso de investigación.

\section{Conclusiones}

El aporte teórico y metodológico de la antropología al trabajo de investigación en bioética permite abordar de modo riguroso el tratamiento de los fenómenos de comportamiento moral, al reconocer al ser humano como producto y productor de la cultura. Es en la sociedad donde los individuos reciben los contextos que les permiten formalizar los fenómenos que perciben: "la sociedad, con sus instituciones, valores, conceptos y lengua es sociológicamente anterior a sus miembros particulares, que sólo se convierten en hombres a través de la educación o adaptación a una sociedad determinada" (11).

El referente social (espacio-tiempo) es necesario para que el estudio de los fenómenos del comportamiento humano pueda ser entendido desde la lógica del sujeto como constructor de significados. Siendo el objeto de estudio de la bioética el comportamiento moral, resulta enriquecedor el aporte de una disciplina como la antropología, que ha desarrollado una serie de técnicas y métodos para la obtención de información, controlando los sesgos propios de la ecuación personal, así como un cuerpo teórico que permite construir mecanismos interpretativos acerca del comportamiento social.

Pero el hombre es también, además de un ser social, un ser individual, que adopta decisiones sobre la base de construcciones respecto de la realidad y de cálculos estratégicos sobre respuestas esperadas, por lo que habría que entenderlo no como un ser determinado, sino como un proceso dinámico. El estudio de la identidad, entendida como constructo, ayuda a entender la diversidad en la homogeneidad.

Las respuestas fáciles de aplicación mecánica, basadas en la existencia de principios indemostrables, no hacen sino ocultar la falta de rigor en el análisis de los fenómenos, cuando no intereses inconfesados que intentan imponer un modo de interpretar la realidad.

\section{Referencias}

1. Ingarden R. Lo que no sabemos de los valores. Madrid: Ediciones Encuentro; 2002.

2. Moreno Villa M. El hombre como persona. Madrid: Caparrós Editores; 1995: 19.

3. Gómez Pin V. La dignidad: lamento de la razón repudiada. Barcelona: Paidós; 1995: 14.

4. Barfield T, Schussheim V. Diccionario de Antropología. México: Siglo Veintiuno Editores; 2000.

5. Bourdieu P. El sentido práctico. Madrid: Taurus Ediciones; 1991.

6. Pérez Argote A. La identidad colectiva: una reflexión abierta desde la sociología. Madrid: Revista de Occidente; 1986.

7. Malinowski B. Los Argonautas del Pacifico Occidental. Barcelona: Península; 1995: 24.

8. López Coira M. La influencia de la ecuación personal en la investigación antropológica o la mirada interior. En: Cátedra M, (ed.) Los españoles vistos por los antropólogos. Madrid: Jucar; 1991.

9. Junker BH. Introducción a las ciencias sociales. El trabajo de campo. Buenos Aires: Marymar; 1972.

10. Genovés S. Ciencia y trascendencia. México: UNAM, Instituto de Investigaciones Antropológicas; 1995.

11. Dumont L. Ensayos sobre el individualismo. Madrid: Alianza Editorial; 1987: 86.

Recibido: 1 de septiembre de 2006.

Aceptado: 12 de diciembre de 2006. 